\title{
CONTINUING CRACK-TIP DEFORMATION AND FRACTURE FOR PLANE-STRAIN CRACK GROWTH IN ELASTIC-PLASTIC SOLIDS
}

\author{
J. R. Rice and E. P. Sorensen ${ }^{\dagger}$ \\ Division of Engineering, Brown University, Providence, RI 02912, U.S.A.
}

(Received 6 March 1978)

\begin{abstract}
ANALYSIS of the deformation field consistent with a Prandtl stress distribution travelling with an advancing plane-strain crack reveals the functional form of the near tip crack profile in an elastic-plastic solid. The crack opening $\delta$ is shown to have the form $\delta \sim r \ln$ (const. $/ r$ ) at a distance $r$ from the tip. This observation coupled with data generated from finite element investigations of growing cracks in small-scale yielding permits the construction of a relation characterizing the deformation at an extending crack tip. A ductile crack-growth criterion consisting of the attainment of a critical opening at a small characteristic material distance from the tip is adopted. Predictions of the stability of a growing crack for both small-scale yielding specimens and those subject to general yielding are discussed.
\end{abstract}

\section{INTRODUCTION}

THE DISTRIBUTIONS of stress and strain accompanying stationary cracks in various elastic-plastic materials and various modes of loading are available from analytical and numerical solutions, e.g. RICE (1976). These investigations, together with extensive experimental studies, e.g. BEGLEY and LANDES $(1972,1976)$ and GREEN and KNOTT (1975), have led to the characterization of the onset of crack growth in terms of a critical value of the $J$-integral, $J_{\text {Ic }}$ for plane strain, or equivalently a critical value of the crack opening displacement, $\delta_{\mathrm{Ic}}$, assuming that certain minimum specimen size limits are exceeded. These parameters are useful in quantifying the onset of fracture; however, the question of whether such fracture is initially stable or catastrophic remains unresolved and there is evidence for stable crack growth in many ductile metals, e.g. Clarke et al. (1976), Griffis and Yoder (1976), Paris et al. (1977) and Clark et al. (1978).

The analytical investigation of extending cracks is difficult mathematically, although some progress has been made in the Mode III or anti-plane strain case (MCClintock and Irwin, 1965). For the tensile or Mode I case, only the general functional form of the near tip singular strain field is known. This was given by RiCE (1968a) in an analysis of steady-state plane-strain crack growth in elastic ideallyplastic solids, and his analysis has been extended in different ways by CHEREPANOV

$\dagger$ Present affiliation: Mathematics Department, General Motors Research Laboratories, Warren, MI 48090, U.S.A. 
(1974) and Rice (1975) for general non-steady growth. Also, Amazigo and Hutchinson (1977) give the form of the singular crack-tip strain field for steady-state growth in isotropically-hardening solids with flow strength increasing linearly with strain. Results in the ideally-plastic case indicate that the strain at the crack tip is governed by a ( $\ln r)$-singularity as opposed to the stronger $(1 / r)$-singularity in the stationary case. This reduced strain concentration originates in the irreversible straining in the plastic zone surrounding the crack tip so that complete refocusing of the strain at the tip of the extended crack tip is prevented. In this sense, elastic strains are destabilizing, since their reversibility promotes additional straining at an advancing crack tip (DrUCKER and RICE, 1970). The reduced strain concentrations accompanying extending cracks partly explain the phenomenon of stable crack growth.

Numerical investigations of stable crack growth include the plane-strain studies of KFouri and Miller (1976) and SOREnSEN (1977) and the plane stress studies of DE Koning (1977) and ANDERsSON (1973). These latter two investigators conclude that a possible parameter for the characterization of continuing fracture is the crack-tip opening angle, the angle between the separating crack surfaces behind the extending crack tip. The difficulty with this parameter, as shown by RICE (1975), is that an extending crack in an elastic-plastic material exhibits a displacement profile proportional to $r \ln r$ (where $r$ is measured from the crack tip) and this implies a vertical tangent at the crack tip with a correspondingly ambiguous definition of the crack-tip opening angle. However, this objection is not present at a small but finite distance away from the crack tip where perhaps a meaningful definition of this parameter may be made.

KFOURI and Miller (1976) propose a Griffith-like critical separation energy as a criterion for continuing fracture. This quantity is defined as the work per unit depth of specimen expended in extending a crack by a distance $\Delta l$ divided by that distance. Although intuitively appealing, such a separation energy is zero for infinitesimal crack advance when the stress level at the advancing crack tip is finite (RICE, 1966). A more serious shortcoming of this quantity is its failure to account for strain accumulations at the crack tip as the critical separation energy is traction controlled. In the limiting case of an elastic perfectly-plastic material at limit load, similar values of the critical separation energy may be obtained for crack advance under the same stress state but vastly different levels of near tip straining. For strain-dominated fracture mechanisms, such as void growth and localization of shear, it seems inappropriate to employ a fracture parameter which does not reflect the strain state at the crack tip.

The recent experiments of Griffis and Yoder (1976) and CLARKE et al. (1976) indicate that under monotonic loading the (nominal) $J$-integral continues to rise monotonically with crack advance. GREEN and KNOTT (1975) report similar increases of the (nominal) crack opening displacement with crack extension. These observations have prompted attempts to characterize stable crack growth using $J$ and $\delta$ as macroscopically mcasurablc quantitics, c.g. Paris et al. (1977) and Clark et al. (1978). The following discussion lends support to this kind of characterization but also suggests limitations to the universality of the stable growth representations thus obtained. 


\section{A Relation for Characterizing Continuing Fracture}

SORENSEN (1977) presents finite clement solutions for crack advance under wellcontained, small-scale yielding, plane-strain conditions. These solutions simulate crack advance through the finite element grid by the successive relaxation of nodal constraints; the external load is held constant during crack advance and is incremented at constant crack length between crack growth steps of one element each. Typically, during the growth process, the maximum radius of the plastic zone was $8-12$ times the size of the near tip elements (although the plastic zone extends directly in front of the crack only over $2-3$ elements). The material was modelled as an elastic-plastic Mises material satisfying the Prandtl-Reuss flow rule, and strain hardening was modelled by a power-law hardening formulation, such as that used by TRACEY (1976), namely

$$
\frac{\bar{\sigma}}{\sigma_{0}}=\left[\frac{\bar{\sigma}}{\sigma_{0}}+\frac{3 E e^{\mathrm{p}}}{2(1+v) \sigma_{0}}\right]^{N},
$$

where $\bar{\sigma}$ is the equivalent tensile stress, $\sigma_{0}$ is the yield strength in tension, $\bar{e}^{-\mathbf{p}}$ is the equivalent plastic strain, $E$ is the elastic tensile modulus, $v$ is Poisson's ratio and $N$ is the hardening exponent. Numerical results for hardening and non-hardening $(N=0)$ analyses indicate that the stress distribution ahead of an advancing crack tip is essentially invariant when plotted against distance normalized by $\left(K_{\mathrm{l}} / \sigma_{0}\right)^{2}$. Further,

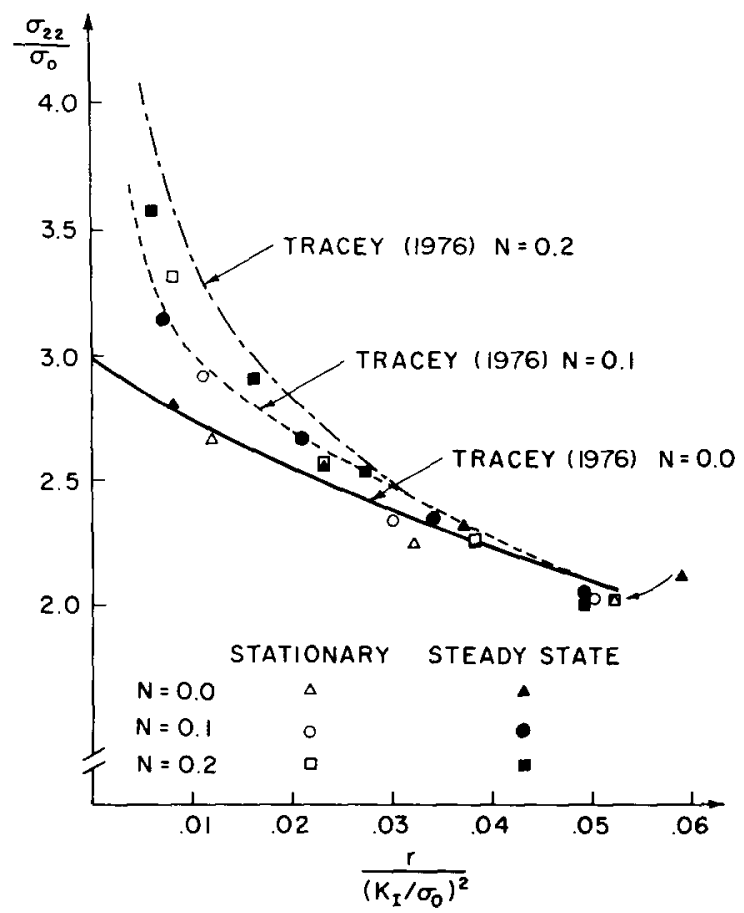

FIG. 1. Tensile stress $\sigma_{22}$ ahead of the crack tip, from Sorensen (1977). Points marked "steady state" correspond to final advancing crack solution. 
this stress distribution is similar to those developed by TRACEY (1976) for monotonically-loaded stationary cracks. Figure 1 presents a plot of the tensile stress ahead of a crack tip before crack growth and following several increments of crack advance (curves taken from TrACEY (1976) are included on the plot). The essentially unchanging stress field (except that given stress levels extend over wider zones of material as $K_{\mathrm{I}}$ is increased) argues for a deformation-dependent fracture criterion, at least when the fracture mechanisms are "strain controlled", to control possible stable crack growth following the initiation of crack advance.

The numerical investigations of SORENSEN (1977) provide crack surface displacements throughout the prescribed loading history. Figure 2 presents cracksurface profiles prior to crack advance and following the final step of crack growth. For the stationary crack, the value of $\delta_{t} /\left(J / \sigma_{0}\right)$ in the non-hardening case (where $\delta_{1}$ is the crack-tip opening displacement, extrapolated to $r=0$ as shown in the figure, and $J$ is equal to $\left.\left(1-v^{2}\right) K_{1}^{2} / E\right)$ is $0 \cdot 66$. For the hardening cases, we use Hutchinson's (1968) and RICE and ROSENGREN's (1968), (denoted by HRR in the sequel), theoretical result that $\delta \sim r^{N /(1+N)}$ very near the crack tip, and write

$$
\delta=\delta_{1}\left(r / r_{1}\right)^{N /(1+N)},
$$

where $\delta_{1}$ is the opening displacement at the first node behind the crack tip and $r_{1}$ is the position of that node. This extrapolation is shown by the dashed lines on the

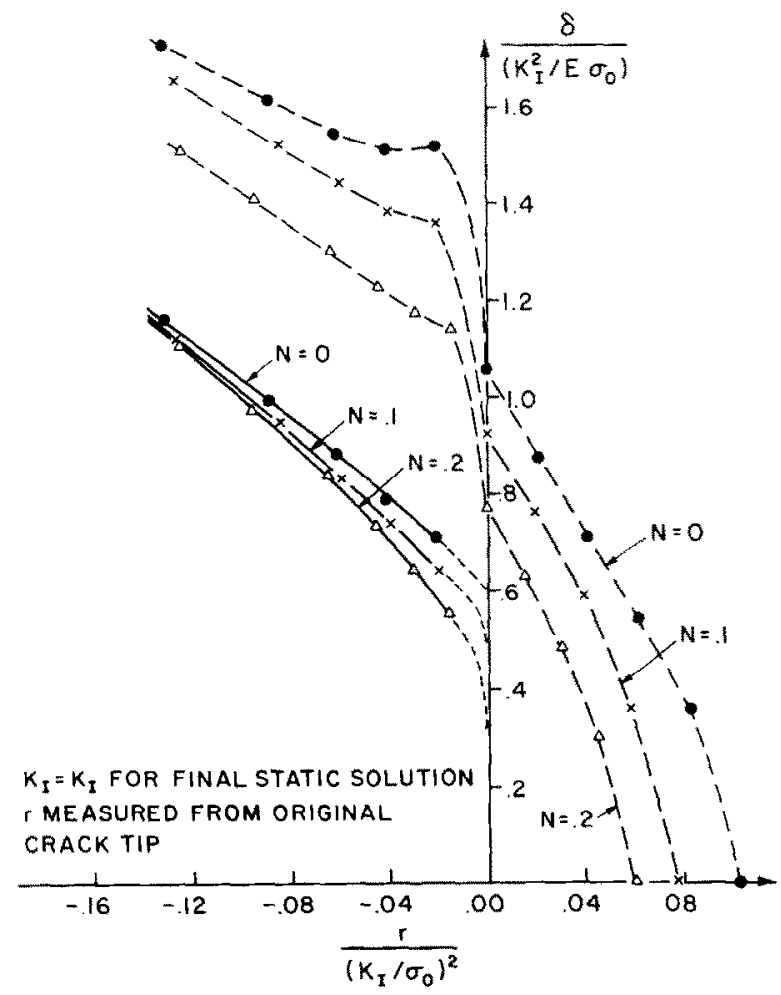

FIG. 2. Crack-surface displacements, stationary and final advancing crack solutions for $N=0.0,0 \cdot 1,0 \cdot 2$, based on SORENSEN's (1977) numerical results. 
figure. Then, following the procedure of TraCEY (1976), we define $\delta_{\mathrm{t}}$ as the opening $\delta$ at the place where a $45^{\circ}$-line drawn back from the crack tip would intersect the deformed crack surface. Thus, we set $r=\frac{1}{2} \delta_{\mathrm{t}}$ in the above equation and solve for $\delta_{\mathrm{t}}$ to obtain $\delta_{t} /\left(J / \sigma_{0}\right)=0.47$ and 0.27 for the two hardening cases $N=0.1$ and 0.2 , respectively. For comparison, TRACEY (1976) presents an approximate relation for $\delta_{t}$, based on his finite element solutions with singularity elements and the $45^{\circ}$-line intersection procedure, viz.

$$
\delta_{\mathrm{t}} \approx 0.49 \frac{K_{\mathrm{I}}^{2}}{E \sigma_{0}}(1+N)\left[\frac{2(1+v) \sigma_{0}(1+N)}{\sqrt{3} E N}\right]^{N},
$$

and PARKS (1975) argues that the factor 0.49 should be approximately 20 per cent higher, at least for the non-hardening case, due to the artificial path-dependence of $J$ that seems (through comparison with a corresponding "deformation theory" solution) to be directly traceable to Tracey's non-hardening singularity element. Values of $\delta_{\mathrm{t}} /\left(J / \sigma_{0}\right)$ obtained from the above expression when Poisson's ratio $v$ is taken equal to 0.3 and $\sigma_{0} / E=1 / 1000$, corresponding to the value used in Sorensen's (1977) analyses, are $0.65,0.47$ and 0.30 for $N=0.0,0.1$ and 0.2 , respectively, and using Parks' correction to the above equation. TRACEY's (1976) analyses employ a small-strain formulation and singular elements, whereas McMEEKING (1977) obtains results for crack-tip openings using a finite-strain formulation. The latter investigator reports values for the non-dimensionalized crack opening displacement between 0.55 and 0.67 in the non-hardening case, between 0.41 and 0.44 for $N=0.1$, and between 0.27 and 0.30 for $N=0.2$. Thus, we conclude that the above estimates of $\delta_{\mathrm{t}}$ based on SURENSEN's (1977) numerical results, namely $\delta_{t} /\left(J / \sigma_{0}\right)=0.66,0.47$ and 0.27 for $N=0 \cdot 0,0 \cdot 1$ and $0 \cdot 2$, respectively, are in reasonably close agreement with the earlier, and presumably more accurate, solutions of Tracey, Parks and McMeeking for monotonic loading of a stationary crack.

Figure 2 also shows the crack profiles after the final steps of crack advance. They seem to be compatible with the logarithmic dependence of displacement on distance $r$ from the tip, as noted by RICE $(1968 \mathrm{a}, 1975)$ for crack extension in an elastic perfectlyplastic material. This logarithmic-displacement dependence is now derived and its consequences explored.

\subsection{Theoretical analysis of incremental deformations at a growing crack tip}

The Prandtl slip-line construction (Fig. 3) is thought to provide the limiting plane-strain stress distribution, $\sigma_{i j}(\theta)$ as $r \rightarrow 0$, for contained plastic yielding at the tip of a sharp crack in an elastic perfectly-plastic Mises material (RICE, 1968b). The theoretical basis is discussed further by RICE and TraCEY (1973) who note that stress fields given by such slip-line constructions become asymptotically valid solutions of the 3-dimensional Mises equations, even in elastically compressible materials, provided that $D_{33}^{\mathrm{p}} / D_{\max }^{\mathrm{p}} \rightarrow 0$ as $r \rightarrow 0$. Here, $D_{i j}^{\mathrm{p}}$ is the plastic rate of deformation tensor and $D_{\max }^{\mathrm{p}}$ represents its maximum value in the plane of straining; the limit of zero is expected since the crack tip is the site of undounded in-plane strain intensification while the plastic strain in the $x_{3}$-direction is bounded by the plane 


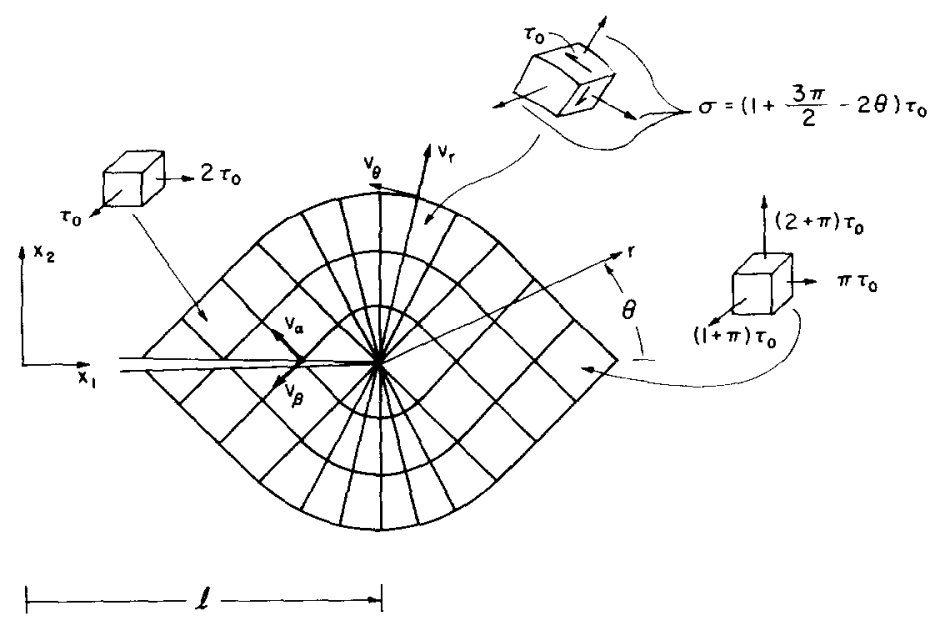

FIG. 3. Prandtl slip-line field stress distribution.

strain constraint and must be the negative of the (bounded) elastic strain. The stress field given by the Prandtl construction arises as the non-hardening limit of the HRR crack-tip singular fields for power-law strain-hardening materials, and it also agrees closely with numerical stress predictions by finite element methods for stationary cracks in elastic-plastic materials (LeVy et al., 1971; RICE and TraCEY, 1973). Further, recent finite element results by SoRENSEN (1977) for growing plane-strain cracks in elastic-plastic materials also suggest the Prandtl field as the limiting cracktip stress state in the ideally-plastic case.

Accordingly, for the present analysis of deformation tields at the tip of a growing crack, we assume that the Prandtl stress-state $\sigma_{i j}(\theta)$ prevails in some small zone near the crack tip and examine the consequences of having this zone move through the material with the advancing crack. As such, our work carries out in detail an integration of the incremental deformation equations as considered in general terms by RICE (1975). A somewhat similar analysis has been given for the case of an elastically incompressible material by CHEREPANOV (1974), in an extension of RICE's (1968a) steady-state growth analysis to non-steady conditions. Note that for the Prandtl field, the deviatoric part, $s_{i j}$, of the stress tensor is such that $s_{33}=0$ and $\sigma_{33}=\sigma$ where $\sigma=\frac{1}{3}\left(\sigma_{11}+\sigma_{22}+\sigma_{33}\right)$ is the mean normal stress.

Within the usual assumptions of the "small strain" approximation in stress analysis, the Prandtl-Reuss flow rule takes the form

$$
D_{i j}=D_{i j}^{\mathrm{e}}+D_{i j}^{\mathrm{p}}=\left[\frac{1+v}{E} \dot{s}_{i j}+\frac{1-2 v}{E} \delta_{i j} \dot{\sigma}\right]+\dot{\Lambda} s_{i j},
$$

where $D_{i j}=\frac{1}{2}\left(\partial v_{i} / \partial x_{j}+\partial v_{j} / \partial x_{i}\right), v_{i}$ is the velocity field, and the distinction between the deformed and undeformed geometry is neglected; and $E$ is the elastic modulus, $v$ Poisson's ratio, and $\dot{\Lambda}$ a non-negative scalar. We observe that

$$
\dot{\sigma}_{i j}=\sigma_{i j}^{\prime}(\theta) \dot{\theta}=\sigma_{i j}^{\prime}(\theta) \sin \theta \dot{l} / r,
$$


where $\dot{\theta}$ is computed at a fixed material-point and the prime denotes a derivative with respect to $\theta$. Further, within the centered-fan zone where the focusing slip-lines allow strain singularities to develop, $\sigma^{\prime}(\theta)=-2 \tau_{0}$, where $\tau_{0}=\sigma_{0} / \sqrt{3}$ is the yield strength in shear. Also, letting $e_{i}(\theta)$ and $h_{i}(\theta)\left[=e_{i}^{\prime}(\theta)\right]$ be unit vectors in the $r$-and $\theta$-directions respectively, one has

$$
e_{i} s_{i j}^{\prime} e_{j}=\left(e_{i} s_{i j} e_{j}\right)^{\prime}-2 h_{i} s_{i j} e_{j}=\frac{d}{d \theta}\left(s_{r r}\right)-2 s_{r \theta}=-2 \tau_{0}
$$

since $s_{r r}=0$ in the fan and $s_{r \theta}=\tau_{0}$.

Thus, when we multiply both sides of (1) by $e_{i} e_{j}$ to form the radial component, we obtain

$$
e_{i} D_{i j} e_{j} \equiv D_{r r} \equiv \frac{\partial v_{r}}{\partial r}=-2(2-v) \frac{\tau_{0}}{E} \frac{\sin \theta}{r} \dot{l},
$$

where $v_{r}$ is the radial velocity.

Hence,

$$
v_{r}=2(2-v) \frac{\tau_{0}}{E} \sin \theta i \ln \left(\frac{\bar{R}}{r}\right)+f^{\prime}(\theta)
$$

where $\bar{R}$ is introduced as some effective cut-off radius to the logarithmic term and the function $f^{\prime}(\theta)$ describes the velocity distribution at $r=\bar{R}$. By noting that $s_{11}+s_{22}=0$ in the fan, one has also from (1) that

$$
D_{11}+D_{22} \equiv \frac{\partial v_{r}}{\partial r}+\frac{v_{r}}{r}+\frac{1}{r} \frac{\partial v_{\theta}}{\partial \theta}=-4(1-2 v) \frac{\tau_{0}}{E} \frac{\sin \theta}{r} i
$$

From this result, we can determine $v_{\theta}$ as

$$
v_{\theta}=-2(2-v) \frac{t_{0}}{E}\left(\frac{1}{\sqrt{2}}-\cos \theta\right) i\left[-\frac{3 v}{2-v}+\ln \left(\frac{\bar{R}}{r}\right)\right]-f(\theta)+g(r),
$$

where $g(r)$ is an arbitrary function of integration.

Since $\sigma_{i j}^{\prime}(\theta)=0$ in the "constant stress" zones of the Prandtl field, where both sliplines are straight, the elastic strain rates vanish in those zones and (1) reduces to $D_{i j}=\dot{\Lambda} s_{i j}$. This implies that the normal components of $D_{i j}$ relative to the slip-lines vanish, and hence the velocity component in the direction of a given slip-line is uniform along the length of that line. Thus, the function $g(r)$, giving $v_{\theta}$ on $\theta=\pi / 4$, must be finite at the crack tip for, otherwise, an unbounded velocity would be propagated along the slip-line at the front border of the centered-fan zone below the crack tip.

We note that a velocity field of the type described by the terms with $f(\theta)$ and $g(r)$ in the expressions for $v_{r}, v_{\theta}$ is of the same form as that which describes opening of a stationary crack (RICE, 1969b); and thus, as remarked generally by RICE (1975), the total velocity field is a combination of these with logarithmically singular terms proportional to $i$. 
Now consider a point on the upper crack surface in the constant stress zone behind the crack tip (Fig. 3). If the totat opening between upper and lower crack surfaces is $\delta$, then $v_{2}=\frac{1}{2} \dot{\delta}$ and

$$
\frac{1}{2} \dot{\delta}=\left(v_{\alpha}-v_{\beta}\right) / \sqrt{2} \text {. }
$$

Here, $v_{\alpha}$ and $v_{\beta}$ are velocities along the slip-lines as shown, so $v_{\beta}=v_{\theta}$ of the fan with $\theta=3 \pi / 4$ and $v_{\alpha}$ is some bounded velocity in the other slip-line direction. Thus, the opening rate at a small distance $r$ behind the crack tip is

$$
\dot{\delta}=4(2-v) \frac{\tau_{0}}{E} i \ln \left(\frac{\bar{R}}{r}\right)+\dot{A},
$$

where $\dot{A}$ embodies all terms except the logarithmically infinite term at the tip. In general, $\dot{A}$ will be linear in $i$ and in rates of parameters describing the external loading. If, for example, the intensity of the external loading is correlated in terms of the $J$ integral value in the deformation field far from the crack tip, then we can write

$$
\dot{\delta}=\alpha \dot{J} / \sigma_{0}+\beta \frac{\sigma_{0}}{E} i \ln \left(\frac{R}{r}\right)
$$

where $\beta=4(2-v) / \sqrt{3}=3.93$ for $v=0.3$, where the additional terms in $\dot{l}$ within $\dot{A}$ have been absorbed into the definition of $R$ (which replaces $\bar{R}$ ), and where $\alpha$ is some dimensionless coefficient. The analysis gives no indication of the value of $R$ but, by dimensional considerations, we expect that it should scale with the size of the cracktip plastic zone.

We observe from (10) significant differences in the kinematics of the opening of stationary versus growing cracks (see also $\operatorname{RICE}(1968 \mathrm{a}, 1975))$. Load increase $(j>0)$ at fixed crack length $(i=0)$ gives a discrete opening displacement immediately at the tip; its full details can be resolved only by a finite deformation analysis of the tip opening, as by Rice and Johnson (1970) and MCMeEking (1977). But, for $i>0$ during load alterations, we may integrate (10) to obtain

$$
\delta=\frac{\alpha}{\sigma_{0}} \frac{d J}{d l} r+\beta \frac{\sigma_{0}}{E} r \ln \left(\frac{\mathrm{e} R}{r}\right),
$$

where e is the natural logarithm base and, for simplicity, we assume that $r$ is small enough so that $\alpha, R$ and $d J / d l$ can be considered as being sensibly constant during crack length changes of size comparable to $r$.

This shows that the crack opening displacement is zero at the crack tip, although the slope $d \delta / d r$ of the opening is logarithmically infinite at the crack tip. The result suggests, therefore, that a reasonably unambiguous definition of a "crack-tip opening angle" can be made only when the $(d J / d l)$-term is large enough to dominate effectively the logarithmic term except for very small values of $r$. Indeed, as RICE (1975) showed, the concept of a crack-tip opening angle is unambiguous only for crack growth in rigid-plastic solids; elastic effects lead to the logarithmic singularity of crack surface slope.

We now examine (10) and (11) as a basis for analysis of SORENSEN's (1977) finite element results for growing cracks. 
2.2 Comparison with finite element predictions of crack-surface displacement

Now, for a stationary crack subject to monotonic load, (10) integrates to the wellknown relation $\delta_{\mathrm{t}}=\alpha J / \sigma_{0}$ for the crack-tip opening displacement and $\alpha$ is a materialdependent non-dimensional number. SORENSEN (1977), in a finite element investigation of slow crack growth, prescribes various steps of crack advance followed by increments of external load at constant crack length and finds that the incremental relation

$$
d \delta=\alpha d J / \sigma_{0}
$$

between nominal crack opening displacement (that at the first node behind the advanced crack tip) and $J$ is applicable for increments of load at constant crack length, with essentially the same $\alpha$ as for the stationary crack solution irrespective of the amount of crack growth. Values of $\alpha$ are within 7 per cent of those corresponding to the stationary crack (see previous discussion) for hardening and non-hardening cases. Thus, increments in the $J$-value associated with the outer elastic field seem, at least for the small-scale yielding case examined, to be related in an almost unique manner to increments in the crack opening very near the tip, although the non-proportional stressing and unloading that accompany crack growth invalidate path-independence of the line integral for $J$ on paths within the crack-tip plastic zone. The generality of this result, e.g. at large-scale yielding, needs to be examined further.

Taking (12) to relate increments of crack opening displacement to increments of external load at constant crack length, the result in (10) suggests correlation of the finite element results for increments of crack-surface displacement accumulated during increments of crack growth with

$$
d \delta=\alpha \frac{d J}{\sigma_{0}}+\beta \frac{\sigma_{0}}{E} \ln \left(\frac{R}{r}\right) d l,
$$

although the second term on the right side of (13) is only rigorously valid as $r \rightarrow 0$ and for perfectly-plastic materials. As such, (13) is at best approximate for hardening materials and for finite $r$, but it exhibits the appropriate behavior as $r \rightarrow 0$ in the nonhardening case and for external loading at constant crack length in the small-scale yielding case.

Integration of (13) at a fixed position $x\left(\equiv x_{1}\right.$ coordinate in Fig. 3$)$ as the crack grows from $l_{1}$ to $l_{2}$ gives

$$
\begin{aligned}
& \delta\left(x, l_{2}\right)-\delta\left(x, l_{1}\right) \\
& =\frac{\alpha}{\sigma_{0}}\left\lfloor J\left(l_{2}\right)-J\left(l_{1}\right)\right]+\beta \frac{\sigma_{0}}{E}\left[\left(l_{2}-x\right) \ln \left(\frac{\mathrm{e} R}{l_{2}-x}\right)-\left(l_{1}-x\right) \ln \left(\frac{\mathrm{e} R}{l_{1}-x}\right)\right],
\end{aligned}
$$

where $l_{2}>l_{1} \geqslant x$ and, to simplify the integration, $R$ is treated as constant during the growth. By taking $l_{1}=x$, so that $\delta\left(x, l_{1}\right)=0$, and treating $d J / d l$ as constant in the growth from $l_{1}$ to $l_{2}$, this last equation gives the opening $\delta$ at a small distance $\Delta l$ bchind the tip as in (11):

$$
\delta=\frac{\alpha}{\sigma_{0}} \Delta l \frac{d J}{d l}+\beta \frac{\sigma_{0}}{E} \Delta l \ln \left(\frac{\mathrm{e} R}{\Delta l}\right),
$$


or, upon rearranging into a form which we will later take as the basis for a fracture criterion,

$$
\frac{\alpha}{\sigma_{0}} \frac{d J}{d l}=\frac{\delta}{\Delta l}-\beta \frac{\sigma_{0}}{E} \ln \left(\frac{\mathrm{e} R}{\Delta l}\right) .
$$

The theoretical value of $\beta=3.93$ based on the Prandtl field analysis has been noted earlier.

A value of $\beta$ may also be estimated from the finite element solutions of SORENSEN (1977) whose small-scale yielding analyses prescribe increments of crack growth at constant external load so that the left side of (14) is zero. The plastic-zone size and, indeed, all geometrical sizes in a small-scale yielding solution for the monotonic loading of a stationary crack are proportional to $E J / \sigma_{0}^{2}$. We expect the parameter $R$ to scale with the size of the plastic zone and we know from SorENSEN's (1977) solutions that the maximum extent of the plastic zone, at a given $J$, is essentially the same for a stationary and a growing crack. Accordingly, we assume in the interpretation of numerical results that $R=\lambda E J / \sigma_{0}^{2}$ where $\lambda$ is a pure number to be determined. Hence, from (14) we expect the numerical result for crack growth at constant $J$ to satisfy

$$
\frac{\delta}{\Delta l} \frac{E}{\sigma_{0}}=\beta \ln \left(\frac{\lambda \mathrm{e} E J}{\sigma_{0}^{2} \Delta l}\right)
$$

when $\Delta l$ is a sufficiently small fraction of the plastic-zone dimension. Figure 4 presents a plot of the incremental displacement $\delta$ of the node immediately behind the crack tip versus $\ln \left(\mathrm{e} E J / \sigma_{0}^{2} \Delta l\right)$ for both hardening and non-hardening analyses of one element growth steps at constant $J$ based on the numerical results of SoRENSEN (1977). Of

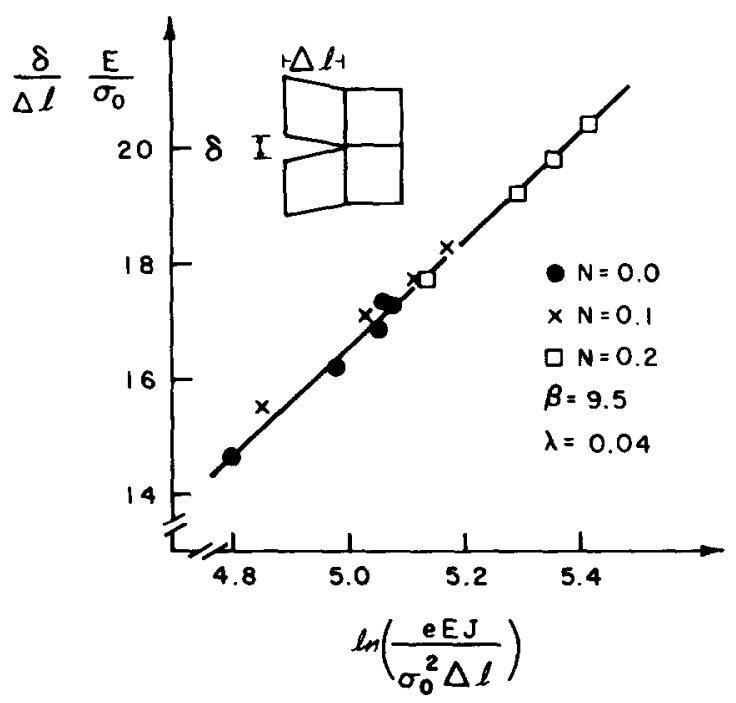

FIG. 4. Correlation of crack opening displacement with crack advance at 1st node behind the crack tip in SORENSEN's (1977) analyses. 
course, for purposes of the present correlation, $\Delta l$ has been set equal to the finite element spacing by which the crack has been advanced. Later, however, we shall wish to identify $\Delta l$ as a characteristic material length. The close correlation with a straight line shown on the plot in Fig. 4, not only for the non-hardening material but also for the hardening cases, lends support to our considerations, but the numerical results are best fit by $\beta=9.5$ (versus a theoretical value of 3.9 ) and $\lambda=0.04$. The $\lambda$-value corresponds to an $R$-value which is approximately $1 / 4$ of the maximum radius of the plastic zone. The discrepancy from the theoretical value of $\beta$ may arise either because of constraints of the finite element grid over the one-element crack growth step or because the manner of unloading the element to simulate growth cannot precisely simulate continuous crack extension. On the other hand, it is well to remember that fracture can be discontinuous on the microscale and this may result in different effective $\beta$-values very near the tip over the size scale of the fracture process zone.

For the second node behind the crack tip, the integration of (13) at constant $J$ can be used as a theoretical basis for another estimate of $\beta$ and $\lambda$. Specifically, choosing the node one element spacing behind the crack tip and letting the crack grow at constant $J$ by one additional element spacing (i.e. $l_{1}-x=\Delta l, l_{2}-x=2 \Delta l$ ), we have an opening $\Delta \delta$ during the growth step given by

or

$$
\Delta \delta=\beta \frac{\sigma_{0}}{E}\left[2 \Delta l \ln \left(\frac{\mathrm{e} R}{2 \Delta l}\right)-\Delta l \ln \left(\frac{\mathrm{e} R}{\Delta l}\right)\right],
$$

$$
\frac{\Delta \delta}{\Delta l} \frac{E}{\sigma_{0}}=\beta \ln \left(\frac{\lambda \mathrm{e} E J}{4 \sigma_{0}{ }^{2} \Delta l}\right),
$$

again assuming that $\Delta l$ is a small fraction of the plastic-zone dimension. Figure 5 shows numerical results from SORENSEN (1977) plotted in this way. The correlation is

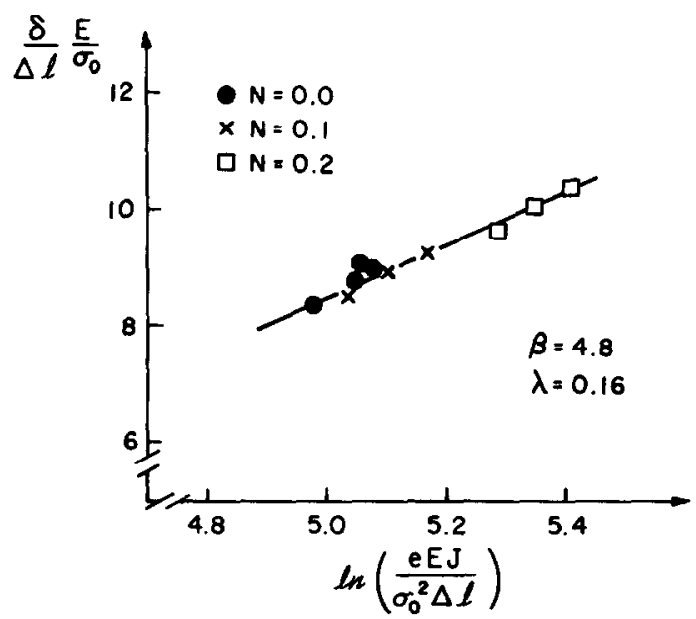

FIG. 5. Correlation of crack opening displacement with crack advance at 2 nd node behind the crack tip in SORENSEN's (1977) analyses. Here, the $\delta$ represents the additional opening in crack growth by one element spacing. 
not as definite; the fits corresponding to the line shown are $\beta=4 \cdot 8$ and $\lambda=0 \cdot 16$. This latter value of $\lambda$ corresponds to a value of $R$ that is approximately equal to the maximum radius of the plastic zone. However, two element sizes may be too far from the crack tip, given the extent of the plastic zone (typically 8-12 elements), for the asymptotic formulation for $\dot{\delta}$ to apply. To resolve fully the near tip openings numerically, it seems necessary to perform an analysis for a substantially finer mesh than the already rather fine mesh used by SORENSEN (1977), so that the correlation of $\delta$ with $\Delta l$ may be definitely investigated. Such an analysis is costly and appears feasible only if performed on the latest generation of high speed computers, with a grid size such that the steps by which the crack advances are smaller than, say, 1/50 of the maximum extent of the plastic zone. (We arrive at this estimate by noting that the finite element results can be expected to be valid only up to $2-3$ elements from the crack tip, yet this distance must be very small compared to overall plastic-zone dimensions for the asymptotic results of (10) to be valid.)

\section{Investigation of a Possible Crack Growth Criterion}

Equation (14) may be used to phrase a fracture criterion which permits investigation of the stability of a growing crack in small-scale yielding conditions. Take the term $\delta / \Delta l$ to be a material-dependent constant, in the sense that to achieve a growth step $\Delta l$ in a material, where the size $\Delta l$ is considered characteristic of the material, it is assumed that a critical value of the crack opening displacement $\delta$ at distance $\Delta l$ must be achieved. This criterion requires that the near crack-tip openings must, in some sense, remain similar in size for continuing crack growth. Indeed, $\delta / \Delta l$ can be thought of as a measure of a crack opening angle. In this sense, our criterion for growth is the achievement of a critical opening angle although, as we have remarked, no such angle can be defined as $r \rightarrow 0$ and our measure of an opening angle is based on the crack surface separation at a fixed, small distance $\Delta l$ behind the tip.

Note that with $R$ proportional to $J$ in (14), i.e. $R=\lambda E J / \sigma_{0}^{2}$, a $J$-level exists at which the $(d J / d l)$-value required for continued growth approaches zero. Denote $J$ at these "steady state" conditions by $J_{\text {ss. }}$. The $J_{\text {ss }}$ can be identified in terms of $\delta / \Delta l$ and $\Delta l$ as

$$
J_{\mathrm{ss}}=\left(\sigma_{0}^{2} \Delta l / \lambda \mathrm{e} E\right) \exp \left[\left(E / \beta \sigma_{0}\right) \delta / \Delta l\right]
$$

and (14) becomes the differential equation

$$
\frac{d J}{d l}=\frac{\beta}{\alpha} \frac{\sigma_{0}^{2}}{E} \ln \left(\frac{J_{\mathrm{ss}}}{J}\right),
$$

describing the $J$-history necessary for continuing stable crack growth. This equation may be integrated from initial crack length $l_{0}$ to current crack length $l$, where a value of $J_{\mathrm{Ic}}$, marking the initiation of growth, is associated with $l_{0}$. Of course, we assume 
$J_{\mathrm{Ic}}<J_{\mathrm{ss}}$ and the two parameters together describe the fracture resistance of the material. The result is

$$
\frac{\sigma_{0}^{2}}{E} \frac{\left(l-l_{0}\right)}{J_{\mathrm{ss}}}=\frac{\alpha}{\beta}\left[E_{1} \ln \left(\frac{J_{\mathrm{ss}}}{J}\right)-\mathrm{E}_{1} \ln \left(\frac{J_{\mathrm{ss}}}{J_{\mathrm{Ic}}}\right)\right],
$$

where $\mathrm{E}_{1}(x)$ is the exponential integral

$$
\mathrm{E}_{1}(x)=\int_{x}^{\infty} \frac{\mathrm{e}^{-u}}{u} d u
$$

The fact that $\left(l-l_{0}\right)$ becomes infinite when $J \rightarrow J_{\text {ss }}$ simply reflects the steady-state condition which permits infinite crack growth by definition. The second term on the right side of (17) is a constant which does not affect the general features of the curves presented on Fig. 6 . These curves are obtained by evaluating $E_{1} \ln \left(J_{\mathrm{ss}} / J\right)$ for various values of $J / J_{\text {ss }}$ and plotting the results for the two $(\alpha, \beta)$-pairs $(0.5,9 \cdot 5)$ and $(0 \cdot 5,3.9)$ corresponding to a representative value of $\alpha$ and the range of $\beta$ obtained from numerical and theoretical considerations. The effect of the large $\beta$-value is to reduce the amount of stable growth crack growth predicted. The curves on Fig. 6 are used as follows. For a particular material, we know $J_{\text {Ic }}$ and $J_{\text {ss }}$, and we enter the curve at a level corresponding to $J_{\mathrm{Ic}} / J_{\text {ss }}$ as at the point so marked. The crack growth $\left(l-l_{0}\right)$ necessary for $J$ to reach any given level is identified as shown on the curve, and the

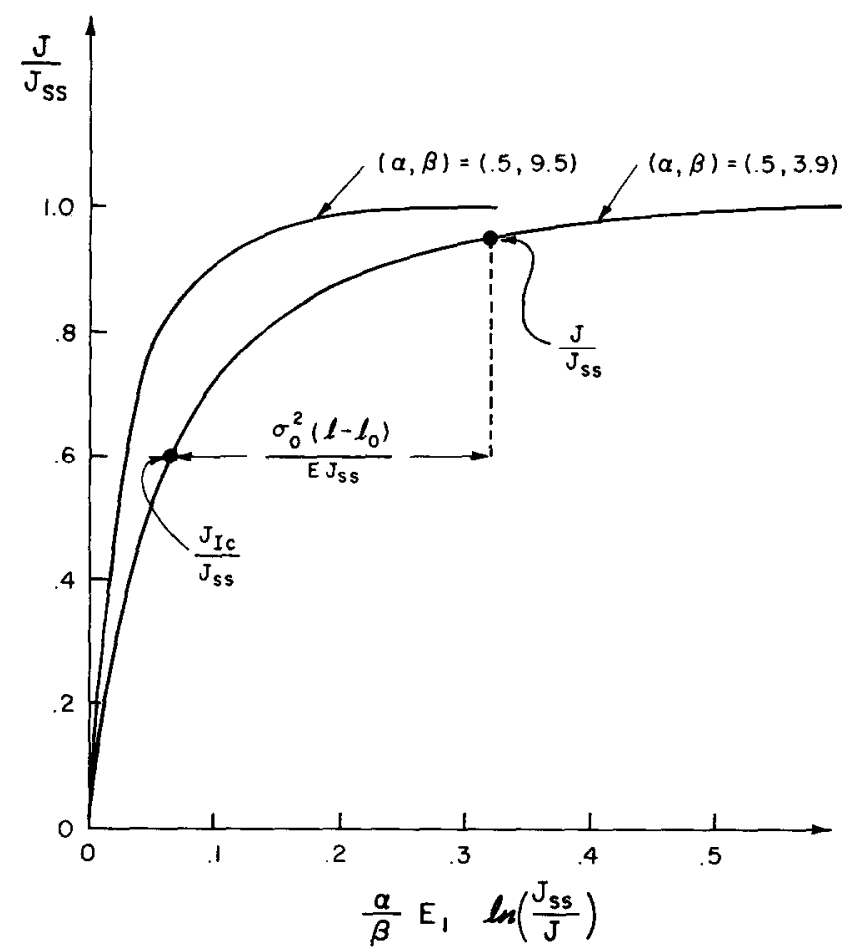

FIG. 6. Crack-growth curves for small-scale yielding as predicted by equation (16). The curves are entered at a point corresponding to $\left(J_{\mathrm{Ic}} / J_{\mathrm{ss}}\right)$, and the dimensionless growth $\left(l-l_{0}\right)$ corresponding to any $\left(J / J_{\mathrm{ss}}\right)$ is measured as shown according to the scale on the horizontal axis. 
dimensionless amount of crack growth, $\sigma_{0}^{2}\left(l-l_{0}\right) / E J_{\mathrm{ss}}$, is measured according to the scale marked on the horizontal axis.

For example, the two points shown correspond to $J_{\mathrm{Ic}}=0.6 J_{\mathrm{ss}}$ and crack growth to a $J$-level of $0.95 J_{\mathrm{ss}}$. Taking $E / \sigma_{0}=300$ and $\delta_{\mathrm{Ic}} \approx J_{\mathrm{Ic}} / 2 \sigma_{0}=25 \mu \mathrm{m}$ as representative of medium to high strength brittle materials, the predicted $\left(l-l_{0}\right)$-value based on the $(\alpha, \beta)$-pair $(0.5,3.9)$ is $6 \mathrm{~mm}$. For the given ratios of $J_{\mathrm{lc}} / J_{\mathrm{ss}}$ and $J / J_{\mathrm{ss}}$, the predicted amount of growth increases proportionally with $E / \sigma_{0}$ and with $\delta_{\mathrm{Ic}}$.

It is remarkable to note that the description of stable crack growth in (15)-(17) and Fig. 6 is of the same mathematical form as deducted by WNUK (1974) from his "final stretch" crack growth criterion based on the Dugdale-Bilby-Cottrell-Swinden plane-stress yield model. In this model, yield is confined to a line ahead of the crack sustaining yield stress $Y$, and Wnuk proposes, essentially, that for crack growth an increment $\delta$ of opening be attained in a small segment $\Delta l$ of the yield zone immediately adjacent to the crack tip. Assuming that $\Delta l$ is small compared to the Dugdale yield-zone dimension $R_{\mathrm{D}}$, where

$$
R_{\mathrm{D}}=\pi \mathrm{K}^{2} / 8 \mathrm{Y}^{2}=\pi E J / 8 \mathrm{Y}^{2}
$$

for small-scale yielding, this criterion becomes (WNUK, 1974)

with

$$
\frac{d R_{\mathrm{D}}}{d l}=\frac{1}{2} \ln \left(\frac{R_{\mathrm{Dss}}}{R_{\mathrm{D}}}\right)
$$

$$
R_{\mathrm{Dss}}=\frac{1}{4 \mathrm{e}} \Delta l \exp \left(\frac{\pi E \delta}{4 Y \Delta l}\right) .
$$

Hence, Wnuk's equations can be put in the forms

$$
\begin{gathered}
J_{\mathrm{ss}}=\left(2 Y^{2} N l / \pi \mathrm{e} E\right) \exp [(\pi E / 4 Y) \delta / \Lambda l], \\
d J / d l=\left(4 Y^{2} / \pi E\right) \ln \left(J_{\mathrm{ss}} / J\right),
\end{gathered}
$$

which may be compared to (15) and (16). Indeed, taking $\beta=3.93$ and $\lambda=0.16$ as suggested earlier, there is close numerical agreement of (18) with (15) when $Y=3 \sigma_{0}$, and taking $\alpha=0.5$ there is close agreement of (19) with (16) when $Y=2.5 \sigma_{0}$. Both values of $Y$ are in a range which might be considered reasonable in order to simulate the plane-strain constraint of the Prandtl field (Fig. 3) in the simple Dugdale model.

Although $J_{\mathrm{ss}}$ enters our description of growth as a limiting value, we note that the attainment of $J_{\mathrm{ss}}$ is not a necessary requirement for unstable fracture. Rather, instability will occur when, under the given conditions of loading, the increment in the applied $J$-value associated with a given virtual increment of crack extension equals or exceeds the increments of $J$ required to continue to meet the fracture criterion. For example, if $Q$ denotes the intensity of some monotonically increasing parameter of loading, the function $J_{\mathrm{A}}(Q, l)$ denotes the applicd $J$-valuc, and the function $J\left(l-l_{0}\right)$ denotes the crack growth criterion, then

$$
J_{\mathrm{A}}(Q, l)=J\left(l-l_{\mathrm{o}}\right)
$$

must be satisfied throughout the growth process. Instability $(d Q / d l=0)$ occurs when

$$
\partial J_{\mathrm{A}}(Q, l) / \partial l=d J\left(l-l_{0}\right) / d l .
$$


The simultaneous meeting of equations (20) and (21) is often represented graphically in terms of a "resistance diagram" (e.g. RICE (1968a)). Thus, the value of $d J / d l$ is the proper measure of stability against rapid fracture. As shown by Fig. 6, this stability measure decreases from its initial value with increasing crack growth, at least under the small-scale yielding conditions envisioned, and rapid fracture occurs when it has fallen to the critical value associated with the particular cracked configuration under consideration.

\subsection{Numerical estimates of the fracture parameters}

We now investigate the relation of the parameters $\delta$ and $\Delta l$ of the fracture criterion to the value of $J_{\mathrm{ss}}$ and to the initial value of $d J / d l$. By its manner of introduction, $\Delta l$ should be of a size comparable to that of the zone of ductile material separation at the crack tip, e.g. the zone in which holes open at particles or inclusions and join together with the macroscopic crack. Now, from various theoretical and experimental studies (Rice and Johnson, 1970; Hahn, Kanninen and Rosenfield, 1972; Green and KnOt, 1976; Rice, 1976; MCMEeKING, 1977), it is known that for the onset of ductile fracture from the tip of an initially sharp crack, the tip opening displacement $\delta$ must attain a size comparable to that of the fracture process zone. Hence, $\delta_{\mathrm{lc}}\left(=\alpha J_{\mathrm{Ic}} / \sigma_{0}\right)$ is an approximate measure of this zone size. The result occurs because the opening determines the size of the region in the material over which plastic strains of order unity occur, and rupture takes place when this region envelops material elements of size comparable to the spacing $X_{0}$ of void nucleation sites. The ratio $\delta_{\mathrm{Ic}} / X_{0}$ is found in the studies cited above to cover a considerable range; and $0 \cdot 5-2 \cdot 0$ is representative. $\mathrm{High}$ values are favored when voids are not readily nucleated from particles; and low values when there is a large volume fraction of particles, or when a second population of void-nucleating particles is activated, and/or when the ductile hole joining process is terminated by a shear localization.

In accord with these observations, we suggest that

$$
\Delta l=\mu \delta_{\mathrm{Ic}}=\mu \alpha J_{\mathrm{Ic}} / \sigma_{0},
$$

where $\mu$ is of order unity and might typically lie between 0.5 and 2 . For the numerical examples to be presented we shall always take $\mu=1$. With the above expression for $\Delta l$, equation (15) yields

$$
\begin{aligned}
J_{\mathrm{ss}} / J_{\mathrm{Ic}} & =(\alpha \mu / \lambda \mathrm{e})\left(\sigma_{0} / E\right) \exp \left[\left(E / \beta \sigma_{0}\right) \delta / \Delta l\right] \\
& \approx 1 \cdot 15\left(\sigma_{0} / E\right) \exp \left[0 \cdot 25\left(E / \sigma_{0}\right) \delta / \Delta l\right],
\end{aligned}
$$

when $\mu=1, \beta=3 \cdot 9, \lambda=0.16$ and $\alpha=0.5$.

If $J_{\mathrm{ss}} / J_{\mathrm{Ic}}$ is extremely large, as favored by a small strength level (i.e. low $\sigma_{0} / E$ ) and/or large opening angle parameter $\delta / \Delta l$, we must conclude that unstable fracture under the presumed small-scale yielding conditions is impossible in reasonably sized spccimcns. Instcad, quite stable crack growth occurs under steeply rising load until fully-plastic conditions are attained, and final rupture instability occurs through a necking-like loss of load-bearing area or perhaps by the type of fully-plastic crack growth instability considered by PARIS et al. (1977). The latter is discussed in 
TABLE 1. Numerical estimates

\begin{tabular}{|c|c|c|}
\hline \multicolumn{3}{|c|}{ For $\delta / \Delta l=0 \cdot 1$} \\
\hline$\sigma_{0} / E$ & $J_{\mathrm{ss}} / J_{\mathrm{Ic}}$ & $T_{\text {init. }}$ \\
\hline 0.0015 & $3.0 \times 10^{4}$ & $80 \cdot 4$ \\
\hline 0.0030 & $14 \cdot 4$ & $20 \cdot 8$ \\
\hline 0.0045 & $1 \cdot 3$ & $2 \cdot 0$ \\
\hline 0.0060 & 0.5 (spontaneous) & $-\cdots$ \\
\hline 0.0075 & 0.03 (spontaneous) & - \\
\hline \multicolumn{3}{|c|}{ For $\sigma_{0} / E=0.0045$} \\
\hline$\delta / \Delta l$ & $J_{\mathrm{ss}} / J_{\mathrm{Ic}}$ & $T_{\text {init. }}$ \\
\hline 0.05 & 0.8 (spontaneous) & - \\
\hline $0 \cdot 10$ & $1 \cdot 3$ & $2 \cdot 0$ \\
\hline $0 \cdot 15$ & $21 \cdot 5$ & 23.9 \\
\hline $0 \cdot 20$ & 346 & $45 \cdot 6$ \\
\hline $0 \cdot 25$ & 5570 & $67 \cdot 3$ \\
\hline \multicolumn{3}{|c|}{ For spontaneous failure (i.e. $J_{\mathrm{ss}} / J_{\mathrm{Ic}} \leqslant 1$ ) } \\
\hline$\sigma_{0} / E$ & $\delta / \Delta l<$ & \\
\hline $0 \cdot 0015$ & 0.03 & \\
\hline 0.0030 & 0.07 & \\
\hline 0.0045 & 0.09 & \\
\hline 0.0060 & $0 \cdot 12$ & \\
\hline $0 \cdot 0075$ & $0 \cdot 14$ & \\
\hline
\end{tabular}

Section 3.2. On the other hand, for high strength materials (i.e. high $\sigma_{0} / E$ ) or for materials requiring only a small opening parameter $\delta / \Delta l$, equation (23) may lead to a result less than unity for $J_{\mathrm{ss}} / J_{\mathrm{Ic}}$. In this case, we conclude that unstable fracture will be spontaneous with the attainment of $J_{\mathrm{Ic}}$ and no stable crack growth will result.

Table 1 shows the results of various estimates of $J_{s s} / J_{l c}$ and of the initial value, calculated from (16) by setting $J=J_{\mathrm{Ic}}$, of a dimensionless resistance parameter

$$
T \equiv\left(E / \sigma_{0}^{2}\right)(d J / d l),
$$

introduced by Paris et al. (1977) and Hutchinson and Paris (1977). Given the tentativeness of our estimates of various parameters from the finite-element solution and of our estimate of $\Delta l$, the table should be regarded as suggesting trends rather than definitive results. First, we consider an opening angle parameter $\delta / \Delta l=0.1$ and show $J_{\mathrm{ss}} / J_{\mathrm{Ic}}$ and $T_{\text {init. }}$ for strength levels ranging from $\sigma_{0} / E$ equal 0.0015 to 0-0075. The first entry is a case for which unstable fracture under contained yielding is practically impossible; the last two are cases for which fracture would be spontaneous, without stable growth. Next, we consider a strength level $\sigma_{0} / E-0.0045$ and show the results for different opening parameters. Finally, the range of opening parameters for which fracture would be spontaneous is given as a function of strength level. 
PARIS et al. (1977) summarize experimental data for plane-strain-like stable crack growth in fully-yielded specimens of a wide variety of ductile materials. They indicate that when growth is correlated in terms of the (nominal) value of $J$, an effectively constant value of $T$ results typically for growth on the order of a few millimeters. The range of $T$-values reported extends from 220 for a cast steel at $149{ }^{\circ} \mathrm{C}$ to 0.15 for a stainless steel at $-296^{\circ} \mathrm{C}$; the majority of $T$-values are in the range $25-125$. While the relation of $T$-values in fully-yielded specimens to those appropriate for well contained yielding remains a point of speculation (see Section 3.2 and Hutchinson and PARIs (1977)), it is interesting to note that the $T$-values listed in Table 1 are within the general range of available data. Also, from (16) with $\alpha=0.5$ and $\beta=3.9$,

$$
J_{\text {ss }} / J_{\mathrm{lc}} \approx \exp \left(0 \cdot 13 T_{\text {init. }}\right),
$$

so that $T$-values on the order of 100 correspond to values of $J_{\mathrm{ss}} / J_{\mathrm{lc}}$ on the order of $10^{5}-10^{6}$. Unstable fracture is then essentially impossible under contained yielding.

CLARK et al. (1978) report similarly the results of fibrous crack growth tests in small, fully-plastic bend specimens of a variety of pressure-vessel steels. They find that the opening displacement at the initial crack tip increases in an approximately linear way with crack growth on the order of a millimeter or so. It is somewhat speculative to identify the slopes which they report in these data with $\delta / \Delta l$ of our fracture criterion based on (14). One might, for example, argue that the observations more closely represent the left side of (14), although the difference will not be great for low strength, highly ductile solids. We report in Table 2 the values of $J_{\mathrm{ss}} / J_{\mathrm{Ic}}$ and $T_{\text {init. }}$ which would be estimated for the fracture properties of these same materials in specimens which arc large cnough to allow well-contained yielding. The materials and conditions of heat treatment, or of preparation in the case of weld deposits, are given; $\delta_{\mathrm{Ic}}$ and $\delta / \Delta l$ are listed directly as the reported tip openings and slope of the opening versus crack length curve at the onset of growth (averaged over approximately $1 \mathrm{~mm}$ growth); $\sigma_{0} / E$ is based on the average of the 0.2 per cent proof stress and ultimate strength when the latter is reported. All tests were at room temperature; less ductile

TABLE 2. Crack growth parameters for specific pressure vessel steels (based on data of CLARK et al. (1978))

\begin{tabular}{|c|c|c|c|c|c|}
\hline Material & $\delta_{\mathrm{Ic}}(\mathrm{mm})$ & $\delta / \Delta l$ & $\sigma_{0} / E$ & $J_{\mathrm{ss}} / J_{\mathrm{Ic}}$ & $T_{\text {init. }}$ \\
\hline $\begin{array}{l}\text { A } 533 \mathrm{~B}\left(1 \mathrm{hr} 670^{\circ} \mathrm{C},\right. \\
\left.36 \mathrm{hr} 620^{\circ} \mathrm{C}\right)\end{array}$ & $0 \cdot 19$ & 0.74 & 0.0024 & $8 \times 10^{30}$ & 555 \\
\hline A533B $\left(4 \mathrm{hr} 650^{\circ} \mathrm{C}\right)$ & $0 \cdot 18$ & 0.20 & 0.0028 & $2 \times 10^{5}$ & $94 \cdot 5$ \\
\hline $\mathrm{HY}-80\left(5 \mathrm{hr} 650^{\circ} \mathrm{C}\right)$ & $\begin{array}{c}0.125 \\
\text { (average) }\end{array}$ & $\begin{array}{c}0.39 \\
\text { (average) }\end{array}$ & 0.0035 & $5 \times 10^{9}$ & 174 \\
\hline HY $-80(20 \%$ prestrain $)$ & 0.045 & $0 \cdot 12$ & 0.0046 & $3 \cdot 6$ & $10 \cdot 0$ \\
\hline $\mathrm{HY}-130\left(2 \mathrm{hr}, 610^{\circ} \mathrm{C}\right)$ & $0 \cdot 06$ & $0 \cdot 13$ & 0.0045 & $7 \cdot 1$ & $15 \cdot 3$ \\
\hline $\begin{array}{l}\text { Weld deposit L } \\
\text { (HY-80 MMA 4-pass) }\end{array}$ & 0.03 & 0.02 & $\begin{array}{c}0.0035 \\
\text { (base material) }\end{array}$ & \multicolumn{2}{|c|}{0.02 (spontaneous) } \\
\hline $\begin{array}{l}\text { Weld deposit N } \\
\text { (HY-100 MMA 4-pass) }\end{array}$ & $0 \cdot 03$ & 0.06 & $\begin{array}{c}0.0037 \\
\text { (base material) }\end{array}$ & \multicolumn{2}{|c|}{0.25 (spontaneous) } \\
\hline
\end{tabular}


behavior would, of course, be expected with lower temperatures, radiation damage, or high loading rates, significantly so when the fracture mechanisms change from fibrous to cleavage.

Evidently, the first three materials listed could not fracture under conditions of contained yielding, and the last two would fracture spontaneously. We recall, from the elastic-plastic solution for small-scale yielding (TrACEY, 1976), that the crack-tip opening displacement is

$$
\delta_{\text {tip }} \approx 3\left(\sigma_{0} / E\right) r_{\mathrm{p}, \max } \approx 0.5 J / \sigma_{0}
$$

where $r_{\mathrm{p}, \max }$ is the maximum radius of the crack-tip plastic zone (occurring at $70^{\circ}$ from the plane prolongation of the crack). Thus, using the reported $\delta_{\mathrm{lc}}$ and $\left(J_{\mathrm{ss}} / J_{\mathrm{Ic}}\right)$ values in the table, we estimate that the pre-strained HY-80 would require $r_{p, \max }$ equal $3.26 \mathrm{~mm}$ at $J_{\mathrm{lc}}$-conditions and $11.7 \mathrm{~mm}$ at $J_{\mathrm{ss}}$-conditions. Further, from Fig. 6 , approximately $21 \mathrm{~mm}$ is the estimated amount of stable crack growth to reach 95 per cent of $J_{s s}$. For HY-130, the corresponding values are $r_{p, \max }$ equal $4.44 \mathrm{~mm}$ at $J_{\mathrm{lc}}$, $31.6 \mathrm{~mm}$ at $J_{\mathrm{ss}}$, and approximately $59 \mathrm{~mm}$ of stable growth to 95 per cent of $J_{\mathrm{ss}}$. The results suggest the general order of sizes required for fracture under well contained yielding in these materials.

It is well to remember that our predictions of the extremely large $\left(J_{\mathrm{ss}} / J_{\mathrm{lc}}\right)$ - and $T$ values in materials of low $\left(\sigma_{0} / E\right)$ and/or high $(\delta / \Delta l)$ result from the assumption that the ductile crack growth process is deformation controlled. That is, it is assumed that a constant opening angle is required for growth irrespective of the spatial extent of the plastic zone. But, as $J$ (or $K_{I}^{2}$ ) increases, the region of large, triaxially elevated stress levels shown towards the left of Fig. 1 envelops a proportionally larger region of the material. In some cases, these high stresses, prevailing over an ever-increasing spatial extent, might serve to nucleate a greater density of microcavities and hence to decrease the opening angle required for continuing growth.

\subsection{Possible implications for fracture under fully-plastic conditions}

For a plane-strain specimen deformed well into the general yielding range, the spatial extent of yielding is limited by the dimension of the remaining ligament. Assuming that the analysis leading to (10), (11) and (14) remains approximately valid and that conditions are met under which the near tip deformation increments can be suitably characterized in terms of some outer-field $J$-value, it would appear that the dimension $R$, reflective of the scale of plastic yielding, would then (by dimensional considerations) be proportional to the ligament size, the coefficient of proportionality depending on specimen type and hardening. The second term on the right side of (14) is then expected to remain near constant during crack advance (at least for $\left(l-l_{0}\right)$ much smaller than ligament size). Thus, equation (14) predicts $d J / d l \approx$ const. This seems to be consistent with the observations of PARIs et al. (1977), mentioned earlier, of an effectively constant $(d J / d l)$-value (based on nominal $J$-values) for fully-plastic

specimens. It also seems consistent with the observation by CLARK et al. (1978) of an approximately linear increase of the nominal $\delta_{\text {tip }}$ with $\left(l-l_{0}\right)$ for small amounts of 
crack growth, if we assume that the nominal $\delta_{\text {tip }}$ and $J$-values remain in approximately the same proportion to one another as for small-scale yielding.

An important issue for the characterization of plane-strain crack growth under a wide range of yielding conditions (e.g. from well contained "small scale" yielding in large specimens to fully-plastic yielding in small specimens of a variety of configurations, such as edge-cracked bend, externally- and internally-cracked tension, etc.) is the clarification of the extent to which some universal, specimenindependent description of the growth process is possible. The matter has received recent attention in relation to the characterization of the onset of crack growth in terms of $J$ and $\delta_{\text {tip. }}$. Such procedures have theoretical justification when there is sufficient strain-hardening that the near tip state is dominated, over a size scale that is large compared to that of the fracture-process zone, by a one-parameter singular strain field of the HRR-type regardless of the specimen type or the extent of yielding. For small amounts of stable crack growth, Hutchinson and PARIs (1977) argue similarly that so long as some appropriately dimensionless measure of $(d J / d l)$ is large, conditions validating the deformation-plasticity basis of the $J$-integral and HRR singular fields will be met everywhere except in some small vicinity of the crack tip. They argue, thereby, that $J$ continues to be a valid measure of the intensity of the outer-field surrounding the fracture zone and suggest on this basis that a universal, specimen-independent relation between $J$ and $\left(l-l_{0}\right)$ may exist as postulated previously by Paris et al. (1977).

A full assessment of this viewpoint is not possible because the theoretical description of crack growth that we have developed in Section 2.1 is based on ideal plasticity and does not include the strain-hardening effects which are essential to the Hutchinson-Paris discussion in terms of one-parameter HRR-fields. We do find, based on limited numerical results by SoRENSEN (1977), that $J$ retains some role as a crack-tip characterizing parameter during growth, in that $\left(\alpha d J / \sigma_{0}\right)$ gives, in $(10)$, the near tip opening $d \delta$ in the absence of further growth, with an approximately constant $\alpha$ irrespective of the amount of growth. However, the last terms in (10), (11) and (14) arise from the contributions of growth $d l$ to the opening. These terms all contain the parameter $R$ which seems to be essentially proportional to the plastic-zone size at small-scale yielding but, as remarked above, to the uncracked ligament dimension in fully-yielded specimens with a Prandtl-like near tip stress field. Hence, the last terms in (10), (11) and (14) suggest at least some dependence of $(d J / d l)$ on the extent of yielding and specimen type.

To investigate this dependence we re-write (14) in terms of the $T$-parameter of PARIS et al. (1977) as

$$
T \equiv\left(E / \sigma_{\mathrm{o}}{ }^{2}\right) d J / d l=\left(E / \sigma_{\mathrm{o}} \alpha\right) \delta / \Delta l-(\beta / \alpha) \ln (\mathrm{e} R / \Delta l)
$$

First, by evaluating $R$ as appropriate for small-scale yielding at conditions near $J_{\mathrm{Ic}}$, we find that the term containing $R$ is typically only a small fraction of $T$ for the smaller $\left(\sigma_{0} / E\right)$ - and larger $(\delta / \Delta l)$-ratios considered in Tables 1 and 2 . Thus, for highly ductile, low strength materials (i.e. large $T$ ), the specimen dependencies would appear to be minimal so long as a Prandtl-like near tip stress state is present in each case. 
Also, by considering different specimen configurations of the same material, denoted by the respective subscripts 1 and 2, one has

$$
T_{2}-T_{2}=(\beta / \alpha) \ln \left(R_{1} / R_{2}\right) \approx 8 \ln \left(R_{1} / R_{2}\right) .
$$

Thus, for example, taking $R_{1}=5 R_{2}$ as representing rather extreme differences between specimens at the onset of growth (say, 1 is a large specimen with well contained yielding and 2 is a small fully-plastic bend specimen with an uncracked ligament dimension that is small compared to the plastic zone of 1 ), we find $T_{2}-T_{1} \approx 13$. This is a small difference for the more ductile materials considered by PARIS et al. (1977) with $T$ in the range 100 or larger. Obviously, it becomes significant for less ductile materials. The observation also suggests that small, fully-plastic bend specimens may yield $T$-values that tend to overestimate the initial $T$-value for cases of well contained yielding. In addition, we expect rounded $J$ versus $\left(l-l_{0}\right)$ curves as in Fig. 6 for contained yielding, but approximately straight-line $J$ versus $\left(l-l_{0}\right)$ curves, with a larger slope, in the fully-plastic specimens.

The preceding discussion of specimen dependencies is not necessarily at variance with BEGLEY and LANDES's (1976) experimental results which indicate very different $(d J / d l)$-values for fully-plastic compact tension and center-cracked specimens of the same material. For the ductile rotor steel which they studied, this discrepancy may be caused by different paths of crack advance; along $45^{\circ}$-lines in the center-cracked panel and straight ahead in the compact tension specimen. The occurrence of $45^{\circ}$ crack growth would seem to mean that conditions in the center-cracked specimens were well removed from those for existence of a unique near-tip HRR singular field, characterized by $J$. Indeed, a recent finite element analysis by MCMEEKING and PARKS (1977) suggests that the HRR-field may not generally result over any significant neartip size scale in fully-plastic center-cracked specimens. But for 6061-T651 aluminum specimens (Professor J. A. Begley (Department of Metallurgical Engineering, Ohio State University) and Dr J. D. Landes (Westinghouse Research Laboratory, Pittsburgh), unpublished private communication) for which crack advance is straight ahead in both specimens, the significantly different $T$-values (approximately 3 for compact tension, and 23 for center-cracked panel) might be explained by the low ductility and different values of $R$ in the different specimens. As discussed by RICE (1976), the region over which the characteristic HRR-singularities dominate the strain field is expected, from consideration of the ideally-plastic limit, to be considerably larger in a bend specimen than in a center-cracked tensile specimen (if, indeed, any such region exists in the latter case (MCMEEKING and PARKs, 1977)), and the parameter $R$ should show a similar trend. Thus, the specimen-dependent $(d J / d l)$ values for aluminum may be consistent with (14) and (26) since $R$ is expected to be much smaller for the center-cracked case. These considerations are, of course, highly speculative but their more detailed examination seems approachable within the framework that we develop here, assuming that suitably mesh-refined numerical calculations are carried out in future work, for fully-plastic specimen geometries as well as for the contained yiclding case remarked upon earlier.

Finally, we remark on the significance which PARIS et al. (1977) attach to the dimensionless crack-growth resistance parameter $T \equiv E(d J / d l) / \sigma_{0}^{2}$ in fully-plastic specimens. By modelling the specimen material as elastic ideally-plastic and 
considering virtual crack extensions under conditions for which the load-point displacement is held fixed, these authors calculate by methods similar to those of RICE (1975) the additional deformations imposed on the fully-plastic uncracked ligament due to the incremental unloading of the elastic regions of the specimen. From this additional deformation they calculate the increment in the (nominal) applied $J_{\mathrm{A}^{-}}$ value, and hence calculate the smallest value of the material resistance $(d J / d l)$ that is consistent with stability against rapid crack extension. The results take the form that, for stability, $T$ must exceed some critical value which depends only on the geometric configuration (typically, on the ratios of the ligament dimension to specimen length and width) but not on the specimen material. PARIs et al. (1977) suggest from experiments on long edge-cracked bend specimens that a stability criterion of this type is consistent with observations of stable versus unstable crack growth under imposed displacement conditions.

\section{CONCLUSIONS}

A relation describing the deformation accompanying extending cracks in elasticplastic solids is obtained from theoretical and numerical calculations. This relation is used as the basis for a fracture criterion for ductile crack advance based on a critical opening displacement at a characteristic near-tip distance. The model suggests a strong dependence of the stability of crack growth on strength level and ductility. Low values of $\left(\sigma_{0} / E\right)$ and/or high values of the required tip opening parameter $(\delta / \Delta l)$ suggest a steeply rising $J$ versus change-in-crack-length relation and an impossibility of unstable crack growth under conditions of well contained yielding. The model also seems consistent with differences in observed features of crack growth under smallscale yielding and fully-plastic behavior. Namely, more stable growth is predicted for the fully-plastic specimens and a nearly constant value of $(d J / d l)$ results in this case with effects of specimen geometry dependent on material ductility. The quantitative aspects of the relation are less certain due to the imprecise nature of the nominal plastic-zone dimension $R$ which is fundamental to the fracture model. To quantify more precisely the nominal plastic-zone dimension, finite element investigations similar to those of SORENSEN (1977) but with a higher degree of mesh refinement in the vicinity of the extending crack tip are desirable, particularly for fully-plastic configurations. In addition, it is well to remember that the theoretical considerations of the present paper are based on the simplest isotropic hardening (or non-hardening) "flow" theory of plasticity. This may not he fully adequate for characterizing deformations due to the strongly non-proportional stressing paths experienced near a growing crack tip.

\section{ACKNOWLEDGMENT}

This study was supported by the U.S. Energy Research and Development Administration (Washington, D.C.) under Contract EY-76-S-02-3084 with Brown University. We are grateful for discussions of our ideas with Professor J. W. Hutchinson (Division of Applied 
Sciences, Harvard University), Mr J. Pan (Division of Engineering, Brown University), Professor P. C. Paris (Department of Mechanical Engineering, Washington University) and Dr C. F. Shih (General Electric Co., Schenectady).

\section{REFERENCES}

Amazigo, J. C. and HutChinson, J. W.

ANDERSSON, $\mathrm{H}$.

BEGLEY, J. A. and

LANDES, J. D.

Cherepanov, G. P.

Clark, G.,

ElSoudani, S. M., FERGUSON, W. G.,

Smith, R. F. and

KNOTT, J. W.

Clarke, G. A.,

ANDREWS, W. R.,

PARIS, P. C. and

SCHMIDT, D. W.

DRUCKER, D. C. and

RICE, J. R.

GREEN, G. and

KNoTT, J. F.

Griffis, C. A. and YODER, G. R.

HAhN, G. T., Kanninen, M. F. 1972 and Rosenfield, A. R.

HutCHINSON, J. W.

HuTCHINSON, J. W. and

PARIS, P. C.

KFouri, A. P. and

MiLLER, K. J.

DE Koning, A. U.

Levy, N., Marcal, P. V.,

OSTERGREN, W. J. and

RICE, J. R.
1977

1973

1972

1976

1974

1978

1976

1970

1975

1976

1976

1968

1977

1976

1977

1971
J. Mech. Phys. Solids 25, 81.

Ibid. 21, 337.

Fracture Toughness, Part II, ASTM-STP-514, p. 1. American Society for Testing and Materials, Philadelphia.

Int. J. Fracture 12, 764.

Mechanics of Brittle Fracture (in Russian), p. 271. Gos. Izdat., Moscow.

Ductile Crack Extension in Pressure Vessel Steels. Conference on Tolcrance of Flaws in Pressurized Components. Institution of Mechanical Engineers, London. 16-18 May 1978.

Mechanics of Crack Growth, ASTM-STP-590, p. 27. American Society for Testing and Materials, Philadelphia.

Engng Fracture Mech. 1, 577.

J. Mech. Phys. Solids 23, 167.

Trans. ASME, J. Engng Materials and Technol. $98,37$.

Ibid. 98, 152.

A. Rev. Mater. Sci. 2, 381.

J. Mech. Phys. Solids 16, $13 . \dagger$

Stability Analysis of J-Controlled Crack Growth. Proceedings of the ASTM Symposium on Elastic-Plastic Fracture (Atlanta. November 1977), ASTM-STP. In press. American Society for Testing and Materials, Philadelphia.

Proc. Inst. Mech. Engrs 190, 571.

Fracture 1977 (edited by D. M. R. Taplin), (Proceedings of the Fourth International Conference on Fracture. University of Waterloo. 19-24 June 1977), Vol. 3, p. 25. University of Waterloo Press.

Int. J. Fracture Mech. 7, 143.

† HutChINSON (1968) and RiCE and RosengREN (1968) are denoted collectively by HRR in the text. 
MCCLintock, F. A. and IRWIN, G. R.

MCMEeKing, R. M.

MCMeEking, R. M. and PARKS, D. $M$.

Paris, P. C., TADa, H., ZAHOOR, A. and ERNST, $\mathrm{H}$.

PARKS, D. M.

RICE, J. R.

Rice, J. R. and Johnson, M. A. 1970

RICE, J. R. and

ROSENGREN, G. F.

Rice, J. R. and Tracey, D. M. 1973
1965

1977

1977

1977

1975

1966

$1968 \mathrm{a}$

$1968 \mathrm{~b}$ 1975

1976

1968
Fracture Toughness Testing and its Applications, ASTM-STP-381, p. 84. American Society for Testing and Materials, Philadelphia.

J. Mech. Phys. Solids 25, 357.

On Criteria for J Dominance of Crack Tip Fields in Large Scale Yielding. Proceedings of the ASTM Symposium on Elastic-Plastic Fracture (Atlanta. November 1977), ASTM-STP. In press. American Society for Testing and Materials, Philadelphia.

A Treatment of the Subject of Tearing Instability, U.S. Nuclear Regularity Commission Report NUREG-0311, August 1977 (available through National Technical Information Service, Springfield, VA).

Some Problems in Elastic-Plastic Finite-Element Analysis of Cracks, Ch. III. Ph.D. Dissertation. Brown University. (Available through University Microfilms, Ann Arbor, MI.)

Proceedings of the First International Conference on Fracture (edited by T. Yokobori, T. Kawasaki and J. L. Swedlow), (Sendai, Japan. September 12-17, 1965), Vol. 1, p. 309. Japanese Society for Strength and Fracture of Materials.

Fracture: An Advanced Treatise (edited by $\mathrm{H}$. Liebowitz), Vol. 2, Mathematical Fundamentals, p. 191. Academic Press, New York.

Trans. ASME 90, Ser. E. J. appl. Mech. 35, 379.

Mechanics and Mechanisms of Crack Growth (Proceedings of Conference organized by the Physical Metallurgy Centre of British Steel Corporation held at Churchill College, Cambridge, 4-6 April 1973), (edited by M. J. May), p. 14. British Steel Corporation, London.

Mechanics of Fracture (edited by F. Erdogan), AMD 19, p. 23. American Society of Mechanical Engineers, New York.

Inelastic Behavior of Solids (Battelle Institute Materials Science Colloquium. Columbus and Atwood Lake, OH. September 15-19, 1969), (edited by M. F. Kanninen, W. F. Adler, A. R. Rosenfield and R. I. Jaffee), p. 641. McGraw-Hill, New York.

J. Mech. Phys. Solids 16, $1 . \dagger$

Numerical and Computer Methods in Structural Mechanics (Proceedings of ONR Symposium), (edited by S. J. Fenves, N. Perrone, A. R. Robinson and W. C. Schnobrick), p. 585. Academic Press, New York. 
186

Sorensen, E. P.

Tracey, D. M.

WNUK, M. P.
J. R. RICE and E. P. SORENSEN

1977 A Numerical Investigation of Plane Strain Stable Crack Growth under Small Scale Yielding Conditions. Proccedings of the ASTM Symposium on Elastic-Plastic Fracture (Atlanta. November 1977). ASTM-STP. In press. American Society for Testing and Materials, Philadelphia.

1976 Trans. ASME, J. Engng Materials Technol. 98, 146.

1974 Trans. ASME 96, Ser. E, J. appl. Mech. 41, 234. 